\title{
A DYNAMIC ADAPTIVE RELAXATION SCHEME APPLIED TO THE EUCLIDEAN STEINER MINIMAL TREE PROBLEM*
}

\author{
FRANÇOIS CHAPEAU-BLONDEAU ${ }^{\dagger}$, FABRICE JANEZ ${ }^{\ddagger}$, AND JEAN-LOUIS FERRIER ${ }^{\ddagger}$
}

\begin{abstract}
The Steiner problem is an NP-hard optimization problem which consists of finding the minimal-length tree connecting a set of $N$ points in the Euclidean plane. Exact methods of resolution currently available are exponential in $N$, making exact minimal trees accessible for only small size problems (up to $N \approx 100$ ). An acceptable suboptimal solution is provided by the minimum spanning tree (MST) which has been shown computable in an $O(N \log N)$ step. We propose here an $O(N)$ process that is able to relax a given initial Steiner tree into a local minimum of its length. This process, based on a physical analogy, simulates the dynamics of a fluid film which relaxes under surface tension forces and stabilizes in an equilibrium configuration minimizing its total length, through purely local interactions. To improve the solution to the Steiner problem, this $O(N)$ relaxation scheme is applied to reduce the length of the MST. This results in a heuristic of a very low $O(N \log N)$ complexity for the Steiner problem, whose performance is shown to compare quite favorably with that of the best available heuristics. Large problem sizes up to $N=10000$ were successfully tackled. A characterization of the asymptotic behavior of the solution of the Steiner problem shows a stabilization to a nonvanishing positive value of the average length reduction achieved over the MST and predicts an average length for the minimal Steiner tree of about $3 \%$ below $0.65 N^{1 / 2}$ for large $N$.
\end{abstract}

Key words. Steiner problem, minimal tree, minimum spanning tree, optimization, relaxation

AMS subject classifications. 90C35, 05C35, 49-04

PII. S1052623494275069

1. Introduction. The Steiner problem is an optimization problem which consists of finding the shortest possible tree connecting a given finite set of $N$ points in the Euclidean plane [1], [2]. A concrete embodiment of this problem is to devise the shortest road network connecting a given set of cities. For this reason, we shall call here cities the points that have to be connected in the Steiner problem. The expression of the solution requires one, in general, to introduce additional points, the Steiner points. The solution of the problem is the minimal Steiner tree, and it is given as a set of linear edges connecting the cities through the medium of the Steiner points. Although very simple to state, the Steiner problem has been proven NP-hard when defined on the usual continuous Euclidean metric. It becomes NP-complete if the Euclidean metric is discretized. The Euclidean Steiner problem is thus at least as difficult as any NP-complete problem [3]. Available algorithms yielding the exact minimal Steiner tree are exponential in $N$ and are now limited to problem sizes of about $N=100$ cities [4]. In order to tackle larger size problems, heuristic algorithms, leading only to suboptimal Steiner trees, have been developed for the Steiner problem. An acceptable suboptimal solution is provided by the MST of the set of cities, which can be computed in an $O(N \log N)$ procedure [41], [31]. The MST also serves as a basis for many heuristics that implement further improvements upon it [26].

In another area of optimization, new algorithms have recently appeared that

* Received by the editors October 3, 1994; accepted for publication (in revised form) September 9, 1996.

http://www.siam.org/journals/siopt/7-4/27506.html

† Faculté des Sciences, Université d'Angers, 2 boulevard Lavoisier, 49000 Angers, France (chapeau@ univ-angers.fr).

$¥$ Laboratoire d'Ingénierie des Systèmes Automatisés, Université d'Angers, 62 avenue Notre Dame du Lac, 49000 Angers, France (ferrier@istia.univ-angers.fr). 
mimic the evolution of physical systems in order to solve optimization problems. Examples are offered by simulated annealing [5], Brownian motion or diffusion [6], neural network models [7], [8], elastic net methods [9], and genetic algorithms [10]. These new approaches have been applied mainly to combinatorial optimization problems, the prototype being the traveling salesman problem [11]. The Steiner problem is not, strictly, a combinatorial optimization problem because the Steiner points that need be introduced for its resolution have positions which, a priori, can vary continuously in the Euclidean plane. This mixed character of the Steiner problem, which exhibits both combinatorial and continuous optimization aspects, adds a special difficulty to its treatment. From an applied standpoint, many practical applications are faced with the Steiner minimal tree problem, as for instance the definition of communication networks or the wiring of electric devices; these can benefit greatly from an efficient resolution of the Steiner problem. In addition, minimal trees can serve as tools for the quantitative characterization of complex sets, branching architectures, or fractally growing structures [12]-[15]. They can also play a role in the representation and processing of data for pattern recognition tasks [16], [17].

In this paper, we propose an $O(N)$ relaxation scheme, inspired from the evolution of a physical system, which is able to relax a given initial Steiner tree into a local minimum of its length. The approach consists of the simulation, in an adapted way, of the dynamics of a fluid film (a soap film) which relaxes under forces due to surface tension, to a configuration that minimizes its total length. When associated with an explicit procedure to construct an initial Steiner tree, the relaxation scheme offers a complete heuristic for the Steiner problem. The relaxation scheme is applied here to an initial tree derived from the MST. The performance of the resulting heuristic is then analyzed and compared for the resolution of Steiner problems with up to $N=10000$ cities.

2. The Steiner problem. For the Steiner problem as stated in section 1, the lengths are evaluated by means of the usual Euclidean distance. We shall use here the term node to indifferently designate a city or a Steiner point as defined in section 1. We define a Steiner tree as a network of linear edges, which forms a connected graph without a cycle, and connects the given set of nodes. The solution of the Steiner problem is given by the Steiner tree of minimal length or minimal Steiner tree. General properties can be established for the minimal Steiner tree of an $N$-city set in the Euclidean plane [1]:

(a) Any angle between two edges has to be at least $120^{\circ}$; consequently every node is connected to the minimal tree by at most three edges.

(b) A Steiner point is connected to the minimal tree by exactly three edges, which together form three $120^{\circ}$ angles.

(c) The number of Steiner points is at most $N-2$.

Exact algorithms have been proposed that determine the minimal Steiner tree for a set of $N$ cities [18]-[25] and [4]. See [26], [27], and [4] for recent surveys. All these exact algorithms have exponential complexity in $N$, making them usable only for small size problems. To date, an upper limit is set in [4] where problems of size up to $N=100$ are exactly solved.

For larger size problems, heuristics have been proposed [28]-[40] that yield only suboptimal Steiner trees with lengths slightly larger than that of the minimal Steiner tree. See also [26] and [27] for a recent survey.

To evaluate the quality of the solution tree produced by a given algorithm it is useful to compare its length with the length of the MST of the corresponding set of 
cities. The MST of a set of $N$ cities is the shortest possible tree formed by connecting the cities with $N-1$ linear edges with no addition of Steiner points. An algorithm is available (see [41] and [31]), which relies on the Delaunay triangulation and the Voronoi diagram of the $N$-city set, to yield its MST in an $O(N \log N)$ procedure. The length reduction $R$ achieved by a given suboptimal Steiner tree over the MST is defined as the ratio

$$
R=\frac{\text { length of MST }- \text { length of suboptimal Steiner tree }}{\text { length of MST }} .
$$

Different upper bounds have been conjectured and tested for the length reduction $R$ [1], [42]. Recently, a general proof has been given [43] that no tree can be found that achieves a length reduction $R$ larger than $1-\sqrt{3} / 2$ (roughly $13.398 \%$ ). However, for actual Steiner problems that were exactly solved, the length reductions obtained were significantly smaller than this theoretical upper bound. In the exact resolution of [22], the maximum reduction reported is $7.55 \%$ for an $N=5$ city problem, and it drops to $5.77 \%$ for an $N=15$ city problem; the average reduction is $3.08 \%$ for $N=5$ and $3.24 \%$ for $N=15$. In view of these results, it seems that for large values of $N$ the average length reduction $R$ of the exact minimal Steiner tree cannot be expected to be larger than about $3.5 \%$. We shall show in the following that the relaxation scheme we propose, when applied to the MST, achieves length reductions that come close to this value.

\section{Description of the relaxation scheme.}

3.1. Physical analogy. The relaxation scheme we propose is based on a physical analogy, which is also presented in [44], and which refers to the following phenomenon. A fluid film with high surface tension (typically a soap film) is hooked between pins (the cities of a Steiner problem) and its width is kept constant. Forces due to surface tension are unit forces exerted along the film. Under these forces the film relaxes to an equilibrium configuration that minimizes the potential energy associated with surface tension (gravity is neglected). In the presence of a constant width for the film, this minimum of energy corresponds to a minimum of the length of the film between the pins.

3.2. Initialization. For application to the Steiner problem, the relaxation scheme we propose has to be provided with an initial Steiner tree that will be relaxed into a local minimum of its length. A Steiner tree, in general, incorporates the set of $N$ cities connected through a certain number of Steiner points. The relaxation scheme operates on a special class of initial Steiner trees that conform with a general property of the minimal Steiner tree. In this condition, the final Steiner tree obtained after relaxation of such an initial Steiner tree will generally provide a good approximate solution to the Steiner problem. This special class is defined by the property that each Steiner point in an initial Steiner tree is endowed with exactly three incoming edges connecting it to other nodes of the initial Steiner tree and possibly to itself in some situations.

When provided with such an initial Steiner tree, the relaxation scheme then consists of the iterative implementation of two basic processes: an evolution process and an interaction process.

3.3. Evolution process. Each Steiner point $S$ in the Steiner tree is allowed to move under the resultant $\vec{F}$ (as defined in Fig. 3) of the three surface tension forces 
exerted by the three edges incoming on $S$. The displacement of $S$ is proportional to $\vec{F}$, with a proportionality coefficient $\lambda$. To improve the stabilization in a suboptimal Steiner tree when $\vec{F}$ decreases while the algorithm converges, the parameter $\lambda$ is gradually reduced to zero with iterations. This prevents the oscillation of a Steiner point $S$ about its equilibrium position in the event that an edge of $S$ with one of its three neighbors has a length approaching zero. The resultant force $\vec{F}$ on $S$ represents the opposite of the gradient (relative to the coordinates of $S$ ) of the sum of the lengths between $S$ and its three neighbors and consequently the opposite of the gradient relative to $S$ of the total length of the tree. The evolution process can thus be viewed as a gradient descent displacing the Steiner point $S$ in the direction yielding, locally, the maximum length reduction to the tree. This gradient descend operates with a fixed topology for the connections between the nodes of the tree. In general, it would terminate early in a poor local minimum of the length of the tree since the topology of connections is not optimized. We shall now introduce the interaction process, which aims at reorganizing the topology of connections to give access to trees with small total length.

3.4. Interaction process. This process consists of the possibility of a reorganization of the connections between two neighboring Steiner points. The interaction process is illustrated in Fig. 1 and takes place as follows. Let us consider a Steiner point $S$ approaching, in the evolution process, another Steiner point $S^{\prime}$ to which it is connected. Before interaction each one of these two Steiner points possesses three connections, among which is the connection $S S^{\prime}$ which will remain untouched in the interaction process. The triplet of connections for $S$ are with the set of nodes $\left\{A_{1}, A_{2}, S^{\prime}\right\}$ and for $S^{\prime}$ with the nodes $\left\{A_{3}, A_{4}, S\right\}$. If, in its approach, $S$ comes within a distance of $T$ from $S^{\prime}$, then an interaction will be allowed. In the interaction process, $S$ considers the eventuality of exchanging one of its neighbors $\left\{A_{1}, A_{2}\right\}$ for one of the neighbors $\left\{A_{3}, A_{4}\right\}$ of $S^{\prime}$. For $S$, to decide this exchange three possible triplets of connections are examined, the current one $\left\{A_{1} S, A_{2} S, S^{\prime} S\right\}$ and two potential ones, $\left\{A_{3} S, A_{2} S, S^{\prime} S\right\}$ and $\left\{A_{1} S, A_{4} S, S^{\prime} S\right\}$. The configuration $\left\{A_{3} S, A_{4} S, S^{\prime} S\right\}$ is not interesting since it represents a simple permutation of the situations of $S$ and $S^{\prime}$ in the tree with no change to its topology of connections. For each of the three possible triplets of connections for $S$, the resultant force on $S$ is computed (as defined in the evolution process of section 3.3). These three forces are compared based on their magnitudes, and the triplet of connections that produced the maximum resultant force, be it the current configuration, is retained for $S$. The resulting, complementary, change of node in the exchange is applied to $S^{\prime}$. This completes the interaction process.

For the relaxation of a fluid film under surface tension forces, a minimal energy at equilibrium is equivalent to a minimal total length. In such a situation where length is energy, the interaction distance $T$ can be interpreted as a physical temperature for the Steiner tree. One can consider that the Steiner points, around their actual positions in the tree, experience a permanent random thermal motion of magnitude $T$. Interaction then takes place when the two clouds of diameter $T$ associated with two Steiner points collide. The temperature $T$ of the Steiner tree is gradually decreased to zero during operation in order to gradually reduce the possibility of interaction and to freeze the tree in a minimum of energy.

Within the physical analogy of the relaxation of a fluid film, both the evolution and interaction processes seek to imitate different aspects of the deformation which minimizes the potential energy or total length of the film. The evolution process alone 

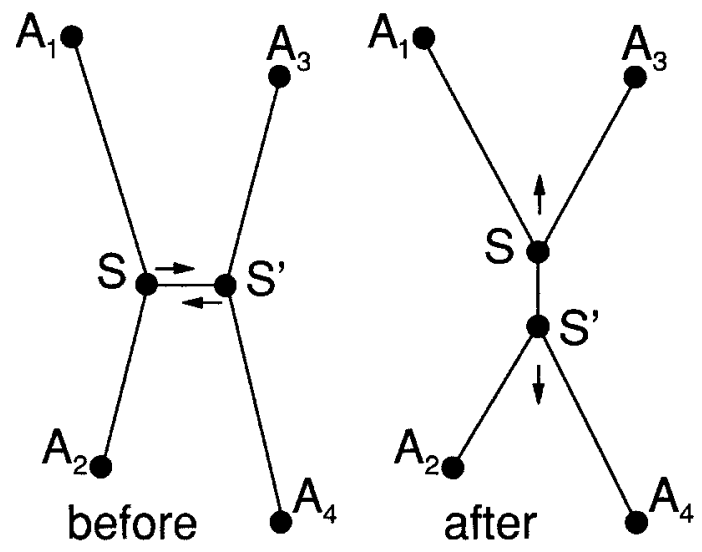

FIG. 1. Interaction process: reorganization of the two triplets of connections of two neighboring Steiner points $S$ and $S^{\prime}$ coming within a distance of $T$ and showing the topology before and after interaction.

displaces the Steiner points along the resultant force, which represents a displacement of the Steiner points in the direction of the maximum length reduction, in the presence of a fixed topology of connections. The interaction process changes the topology of connections to induce locally the maximum resultant force, which represents a change of topology in the direction of the maximum length reduction.

It can be noted that our algorithm bears some similarity with cellular automata [45]. A Steiner point can be considered as an automaton whose state is made up with both the position and the 3-connectivity of the Steiner point. These automata are organized in a network, and they change their state through local interactions with neighbors in the net. The usefulness lies in the collective behavior of the system, which leads, through "microscopic" interactions, to a "macroscopic" configuration realizing a global performance or condition.

4. Application of the relaxation scheme to the MST. When the relaxation scheme is complemented by an explicit procedure to construct an initial Steiner tree, the resulting algorithm offers a complete heuristic for the Steiner problem. We show in the following that a heuristic leading to good suboptimal trees can be obtained when the relaxation scheme is applied to an initial Steiner tree derived from the MST as we now explain.

4.1. An initial Steiner tree derived from the MST. In the MST of the set of $N$ cities, Steiner points are added in order to transform it into the initial Steiner tree that will undergo the relaxation. Figure 2 illustrates how this creation of the Steiner points is performed. In the MST, every city is considered once and processed as follows. For a city with only one incoming edge, no Steiner point is created. For a city with two incoming edges (thus with two neighboring nodes), one Steiner point is created and connected to the city (Fig. 2a). The two neighboring nodes of this city are disconnected from the city and reconnected to the newly created Steiner point. The original city ends up connected to the same pair of neighboring nodes but through the medium of a Steiner point receiving a total of three edges. In a similar way, for a city connected to $n$ neighboring nodes, $n-1$ Steiner points are created. The connections are redistributed between these $1+n+(n-1)$ nodes in order for the original city 
to end up connected to the same $n$ original nodes but through the medium of the $n-1$ Steiner points, each of them receiving a total of three edges. For illustration, this redistribution of the connections is depicted in Fig. 2a for $n=2$, in Fig. 2b for $n=3$, and in Fig. 2c for $n=4$. A simple geometric argument shows that $n$ cannot be larger than 6 , and in practice cities with $n=5$ or 6 incoming edges are very rare in the MST. In practice, the newly created Steiner points are not stacked on top of one another at the location of the original city, but they are distributed around the original city, a very small distance (in comparison with the length scale set by $\lambda$ ) apart from one another and from the city, much like the way they appear in Fig. 2, this in order to avoid a temporary singularity of the type $\overrightarrow{0} / 0$ when computing the resultant force on them for the first time. When every city of the initial MST has been processed once as explained, the MST has become the initial Steiner tree, which serves as the starting tree for the relaxation scheme. For an $N$-city problem, this initialization process creates a total of $N-2$ Steiner points.

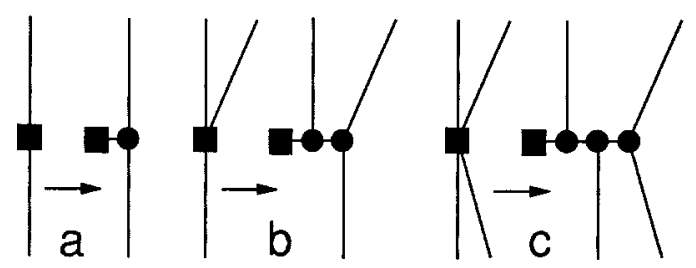

FIG. 2. Initialization process which transforms the MST into an initial Steiner tree: creation of the Steiner points (solid circles) for a city (solid square) of the $M S T$, with $n=2$ in (a), $n=3$ in (b), and $n=4$ in (c), incoming edges.

4.2. Operation of the complete heuristic formed by the relaxation of the MST. The relaxation scheme applied to the initial Steiner tree leads to the algorithm described in Fig. 3.

We want to show that the algorithm of Fig. 3, when applied to the initial Steiner tree derived from the MST, provides a good solution tree to the Steiner problem. In the $N$-city Steiner problems that are considered, the cartesian coordinates of the cities in the Euclidean plane are randomly drawn, with uniform probability, in the unit square $[0,1] \times[0,1]$. In an $N$-city problem, a natural unit of length is provided by $\sigma_{N}=N^{-1 / 2}$. Such a $\sigma_{N}$ gives an image of the average separation between a city and its nearest neighboring city in the unit square for an $N$-city problem. The definition of $\sigma_{N}$ allows one to express the parameters $\lambda$ and $T$ as used in Fig. 3 with numerical values (in units of $\sigma_{N}$ ) that keep the same meaning whatever the size $N$ of the problem. An initial value for $\lambda$ that we found satisfactory and that we retained for operation of the algorithm is $0.02 \sigma_{N}$. A larger initial value for $\lambda$ could make the Steiner tree relax more rapidly to equilibrium, but at the same time useful interactions between Steiner points coming close enough could be missed, leading to an equilibrium Steiner tree of lower quality (of greater length). The initial value for $T$ was selected as $0.15 \sigma_{N}$. Larger values would tend to disorganize the Steiner tree too much, while lower values do not allow enough useful interactions between Steiner points (see Table 1 and its explanation given below).

The schedule that has been used to decrease parameters $T$ and $\lambda$ is a simple one, consisting of a succession of plateaus of descending values. $T$ is first reduced to zero, at constant $\lambda$, in five steps of the same magnitude (equal to one-fifth of the initial value of $T$ ): the first step is taken at iteration $k=100$, the four last steps at iterations 


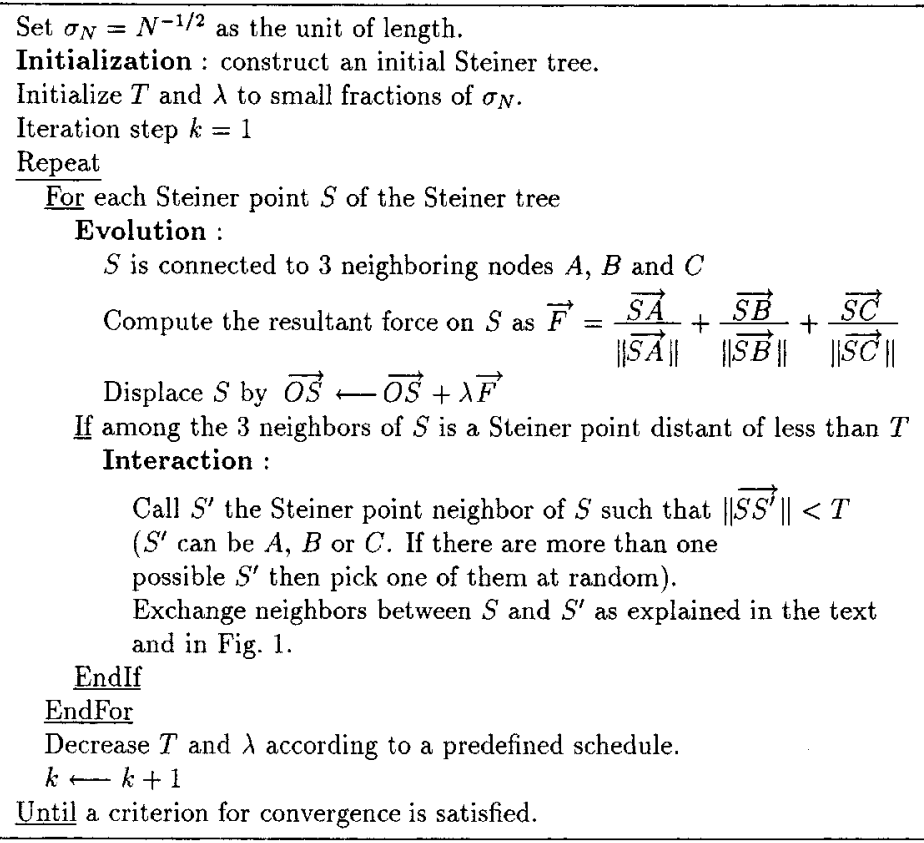

FIG. 3. Complete algorithm for the $N$-city Steiner problem, which results from the application of the relaxation scheme to an initial Steiner tree.

$k=120,140,160$, and 180 , respectively. Then, $\lambda$ is allowed to decay. Starting at iteration $k=200$, the value of $\lambda$ is divided by two each time 20 new iterations have been performed. Such a process is applied until iteration $k=400$ is reached. At this point $\lambda$ has been reduced below $10^{-5} \sigma_{N}$. This sets the criterion of convergence, marking the end of the algorithm. The overall convergence for an $N$-city problem can thus be obtained after an absolute number of iterations of 400, whatever the size $N$ of the city set. We did not address the question of optimizing the schedule for decreasing $T$ and $\lambda$. The value of 400 iterations for convergence can probably be reduced without degrading the quality of the solution tree. What we aimed at with the presented schedule was to have a simple procedure leading to good equilibrium Steiner trees while preserving a complexity of $O(N)$ for the relaxation scheme when performed until convergence.

The importance of allowing, by means of a nonzero temperature $T$, interactions between Steiner points is demonstrated in Table 1. We show in Table 1, for problems of various sizes $N$, the length reduction $R$ (in percents) achieved by the solution tree obtained with different initial values for the temperature $T$. For each condition, the value of $R$ given in Table 1 has been averaged over 100 different problems of size $N$. With a zero initial value for $T$, no interaction is allowed and the length reduction $R$ remains small; as already mentioned $R$ passes through a maximum for an initial $T$ around $0.15 \sigma_{N}$. The role of the interaction process can be interpreted as the ability to select, among the various Steiner tree topologies that are accessible in the vicinity of the initial tree, topologies that can induce local length reductions to the tree.

The relaxation scheme is devised to produce local length reductions to the initial tree through displacements of Steiner points (evolution process) and changes in the topology of connections (interaction process). It can thus be reasonably expected 
TABLE 1

Influence of the initial temperature $T$ (leftmost column) for various problem sizes $N$, and showing the average length reduction $R$ in percents. The initial value of $0.15 \sigma_{N}$ is the one we retained for $T$ in the application of the relaxation scheme to the MST.

\begin{tabular}{|l|c|c|c|}
\hline & $N=50$ & $N=100$ & $N=500$ \\
\hline$T=0$ & 1.762 & 1.665 & 1.663 \\
\hline$T=0.01 \sigma_{N}$ & 2.530 & 2.598 & 2.621 \\
\hline$T=0.15 \sigma_{N}$ & 2.754 & 2.824 & 2.812 \\
\hline$T=0.25 \sigma_{N}$ & 1.894 & 2.003 & 1.913 \\
\hline
\end{tabular}

(prior to the experimental verification that will follow) that the scheme will converge to a good solution tree with reduced length relative to the initial tree. We emphasize that an important property, which justifies that a fixed number of iterations is appropriate for good convergence, is that the parameters $T$ and $\lambda$, which control the local transformations of the tree, scale as $O\left(N^{-1 / 2}\right)$. With this property, what our algorithm basically does is to apply, to the MST in which neighboring nodes are separated by distances of $O\left(N^{-1 / 2}\right)$, a fixed number of local length reductions at the $O\left(N^{-1 / 2}\right)$ scale. This appears to be a reasonable strategy to converge to a solution tree with reduced length relative to the initial MST, without the need to resort to a number of local length reductions that would scale with $N$ instead of being constant. We shall see that this reasonable a priori expectation concerning the convergence is totally confirmed by the experimental evaluation of the algorithm that will follow.

4.3. Algorithmic complexity. The relaxation scheme, formed by the evolution and interaction processes described in section 3, involves only local calculations in the tree at the level of each Steiner point and its three neighbors. For the relaxation of the MST, the initial Steiner tree that is constructed in section 4.1 incorporates a number of Steiner points that is no larger than $N$. It follows then that the relaxation scheme alone, when performed until the convergence obtained after a fixed number of iterations, has a complexity of $O(N)$.

The transformation of the MST into the initial Steiner tree as described in section 4.1 is also $O(N)$.

Now if we turn to the complete heuristic for the Steiner problem that results from the $O(N)$ relaxation of the MST, the overall complexity obviously will depend on the complexity of the determination of the MST.

An algorithm exists (see [41], [31]) that uses the Delaunay triangulation and the Voronoi diagram of the $N$-city set to construct its MST in an $O(N \log N)$ procedure.

In this work, to test the quality of the solution trees provided by our $O(N)$ relaxation of the MST, we constructed the MST with the very simple algorithm consisting of growing the MST by incorporating to it at each step the unconnected point that has the shortest distance with the points already connected in the MST. This method is $O\left(N^{3}\right)$; at the same time it is straightforward to implement, and it allowed us to concentrate our effort on the relaxation scheme which forms the original contribution of this work. However, the MST determined for every $N$-city set is the same, whether computed with the straightforward $O\left(N^{3}\right)$ method we use or the more elaborate $O(N \log N)$ procedure. In the following, we evaluate and compare the quality of the suboptimal trees resulting from the $O(N)$ relaxation of the MST. The association of our $O(N)$ relaxation scheme to the $O(N \log N)$ determination which exists for the MST offers an $O(N \log N)$ heuristic for the Steiner problem that shares the 


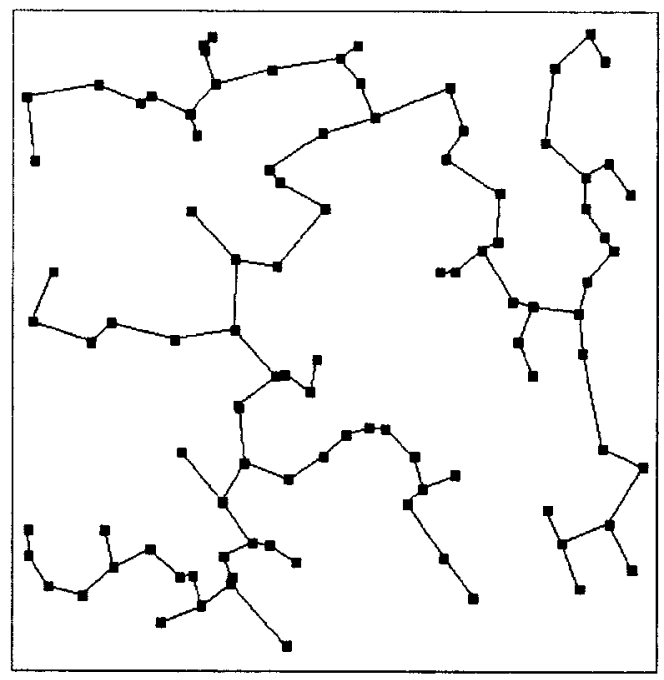

FIG. 4. The MST, with length 6.485 , for a typical $N=100$ city problem. The small black squares represent the 100 cities randomly distributed in the unit square $[0,1] \times[0,1]$.

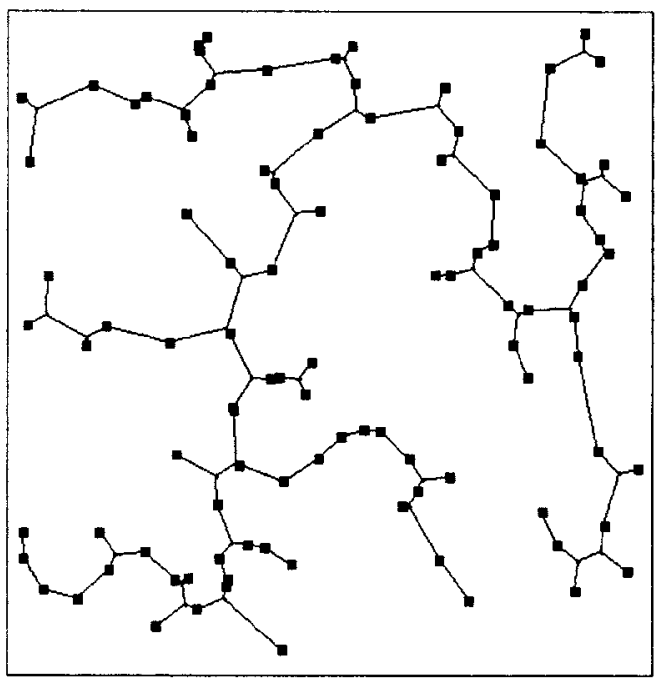

FIG. 5. The suboptimal Steiner tree, with length 6.294, obtained after application of the relaxation scheme to the problem of Fig. 4 and achieving a length reduction of $R=2.945 \%$ over the MST. A Steiner point is located in every place where three edges meet at $120^{\circ}$.

performance we report in the following.

We experimentally verified that the relaxation procedure we propose, by itself, requires a computer time which is, as expected, linear in $N$. When run on an Intel 486 processor with $33 \mathrm{MHz}$ clock, typical computer times for the complete relaxation procedure alone up to convergence (not including the initialization process that computes the MST) are 4 seconds for $N=100,20$ seconds for $N=500,40$ seconds for $N=1000$, 200 seconds for $N=5000,400$ seconds for $N=10000$, and with a dispersion among different runs being less than $2 \%$. 
4.4. Experimental conditions. For illustration of the method, Fig. 4 shows, for a typical Steiner problem with $N=100$ cities, the MST (of length 6.485) which serves both as a starting point to construct the initial Steiner tree and as a reference to evaluate the reduction in length reached by the suboptimal Steiner tree.

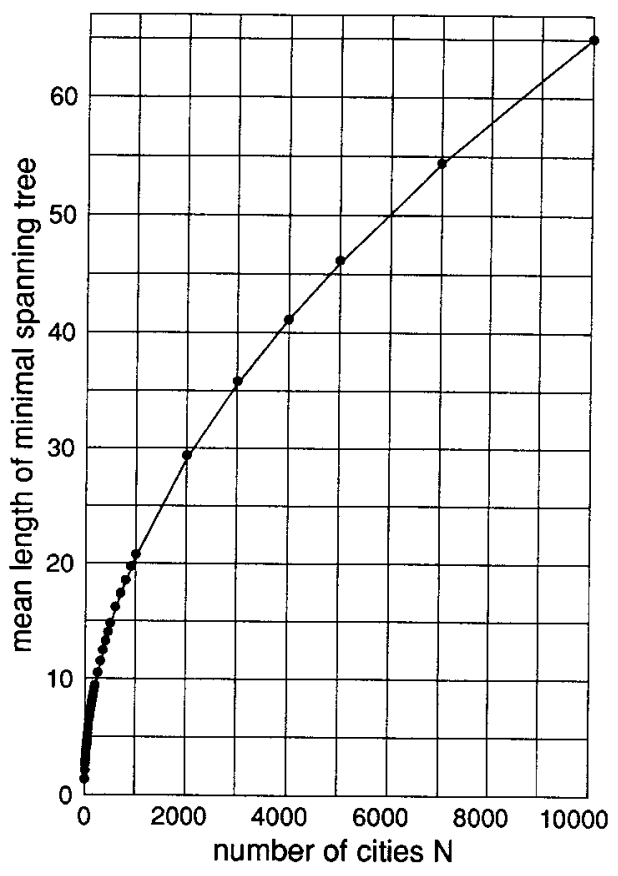

FIG. 6. Mean length of the MST as a function of the size $N$ of the city set, and fitted to a law of the form $0.65 N^{1 / 2}$ (solid line) with a correlation coefficient better than 0.99 .

We then show, in Fig. 5, the suboptimal Steiner tree (of length 6.294) obtained after convergence of the relaxation scheme and achieving here a length reduction of $R=2.945 \%$.

With this method that relaxes the MST, we have performed resolution of Steiner problems with sizes up to $N=10000$ cities. For each tested size $N$, many different problems were generated by random selection of the $N$ cities as explained in section 4.2 , and in order to form a statistical ensemble $\Omega_{N}$ of problems with a given size of $N$ cities. Statistics were then performed over $\Omega_{N}$ which yielded the following quantities:

i) for the MSTs constructed over the $N$-city problems of $\Omega_{N}$ : the mean and standard deviation for their length distribution;

ii) for the suboptimal Steiner trees obtained after application of the relaxation scheme for the $N$-city problems of $\Omega_{N}$ : the mean, standard deviation, and minimum and maximum values of the length reduction $R$.

The evolution of these quantities was then studied as a function of the number of cities $N$ in the Steiner problems. For the statistics, $\operatorname{card}\left(\Omega_{N}\right)$, the cardinality of $\Omega_{N}$ (the number of problems in $\Omega_{N}$ ), was chosen as $\operatorname{card}\left(\Omega_{N}\right)=10^{4} N^{-1 / 2}$ for $N \leq 300$, $\operatorname{card}\left(\Omega_{N}\right)=10^{3} N^{-1 / 2}$ for $300<N \leq 1000$, and $\operatorname{card}\left(\Omega_{N}\right)=5$ for $N>1000$.

In these conditions for performing the statistics, Fig. 6 shows the mean of the length of the MST as a function of $N$. Table 2 displays typical values evaluated for the mean and standard deviation of this length. The data of Fig. 6 and Table 2 give 
TABLE 2

Mean and standard deviation for the length of the MST for various problem sizes $N$.

\begin{tabular}{|l|c|c|c|c|c|c|c|}
\hline$N$ & 10 & 50 & 100 & 500 & 1000 & 5000 & 10000 \\
\hline mean & 2.092 & 4.825 & 6.736 & 14.826 & 20.776 & 46.174 & 65.028 \\
\hline st. dev. & 0.281 & 0.225 & 0.217 & 0.210 & 0.199 & 0.145 & 0.138 \\
\hline
\end{tabular}

an image of the (low) dispersion of the results in the averaging procedure over the statistical ensembles $\Omega_{N}$. We were able to fit the variation of the mean length of the MST to a law of the form $0.65 N^{1 / 2}$ with a correlation coefficient better than 0.99 .

The quality of the solution trees we obtained for the Steiner problems of the statistical ensembles $\Omega_{N}$ is illustrated by the data in the last row of Tables 3 and 4 and in Fig. 7.

5. Evaluation and comparison. In Table 3, the quality of the suboptimal Steiner trees resulting from the relaxation of the MST is compared with that of the solution trees yielded by other resolution methods for the Steiner problem.

As a basis for comparison, we selected

- the exact method of [22], which offers results up to $N=15$, knowing that for the exact resolutions extended up to $N=100$ in [4] quantitative data that would fit into our comparison were not available;

- the $O(N \log N)$ heuristic of [31], which represents, among the efficient heuristics, the one with the smallest algorithmic complexity;

- two heuristics of [36] and [37], which represent, among the efficient heuristics, the ones that generally yield the shortest suboptimal trees. No exact algorithmic complexities are derived in [36] or [37] for these two methods, but estimations are proposed, $O\left(N^{1.317}\right)$ and $O\left(N^{2.19}\right)$, that result from the average computation time on a Cray X-MP/28.

Table 3 gives, for all these different methods, the maximum value, the mean and the standard deviation of the length reduction $R$, and the number of problems of size $N$ that were considered for the statistics.

For the maximum length reduction $R$ in Table 3, it can be noticed that, in every condition, the best maximum was always found by our relaxation of the MST. This is certainly because we explored much larger populations of problems. Over the more than 20000 problem instances that we solved in this study, the maximum $R$ that we report come close $(11.909 \%$ for $N=5)$ but always conform with the theoretical upper bound of $13.398 \%$ established in [43].

When compared with the $O(N \log N)$ heuristic of [31], our approach leads in general to solution trees of better quality. This applies except for the mean $R$ in the case $N=30$ and in the limit case $N=10$. However, in this last condition the mean length reduction of [31] is found larger than that of the exact method of [22], and it is also the case with the heuristics of [36] and [37]. As there is no possibility that a heuristic yields better results than an exact method, we suggest that the mean value of $R$ for $N=10$ in [31], as well as in [36] and [37], obtained by averaging over a small population of problems and associated with a relatively high standard deviation, is marked with statistical fluctuations. The heuristic proposed in [31] is tested therein up to $N=50$. For increasing $N$ approaching $N=50$, this heuristic of [31] seems to entail a steady decay for the mean $R$, while our resolution (and that of [36] and [37]) maintain a mean $R$ constantly above $2.710 \%$. This saturation to a constant value as $N$ increases, rather than a steady decay of the mean $R$, is a trend that will appear 
TABLE 3

Maximum value, mean and standard deviation of the length reduction $R$ (in percents) achieved over the MST, and the number of problems tested with different algorithms for the resolution of the $N$-city Steiner problem.

\begin{tabular}{|c|c|c|c|c|c|c|c|c|c|}
\hline & $N$ & 5 & 10 & 15 & 20 & 30 & 40 & 50 & 60 \\
\hline $\begin{array}{l}\text { Exponential } \\
\text { exact method of [22] }\end{array}$ & $\begin{array}{l}\max . R \\
\text { mean } R \\
\text { st. dev. } \\
\text { nb. pb. }\end{array}$ & $\begin{array}{l}7.55 \\
3.08 \\
25\end{array}$ & $\begin{array}{c}5.89 \\
3.00 \\
25\end{array}$ & $\begin{array}{c}5.77 \\
3.24 \\
\\
25\end{array}$ & & & & & \\
\hline $\begin{array}{l}O(N \log N) \\
\text { heuristic of [31] }\end{array}$ & $\begin{array}{l}\max . R \\
\text { mean } R \\
\text { st. dev. } \\
\text { nb. pb. }\end{array}$ & & $\begin{array}{c}6.847 \\
3.173 \\
2.09 \\
15\end{array}$ & & $\begin{array}{c}4.227 \\
2.333 \\
0.70 \\
15\end{array}$ & $\begin{array}{c}4.554 \\
2.769 \\
0.89 \\
15\end{array}$ & $\begin{array}{c}4.014 \\
2.663 \\
0.64 \\
15\end{array}$ & $\begin{array}{c}3.443 \\
2.568 \\
0.57 \\
15\end{array}$ & \\
\hline $\begin{array}{l}O\left(N^{1.317}\right) \\
\text { heuristic of }[36]\end{array}$ & $\begin{array}{l}\max . R \\
\text { mean } R \\
\text { st. dev. } \\
\text { nb. pb. }\end{array}$ & & $\begin{array}{c}6.168 \\
3.138 \\
1.863 \\
15\end{array}$ & & $\begin{array}{c}4.737 \\
3.015 \\
1.008 \\
15\end{array}$ & $\begin{array}{c}4.752 \\
2.868 \\
0.721 \\
15\end{array}$ & $\begin{array}{c}4.174 \\
3.024 \\
0.631 \\
15\end{array}$ & $\begin{array}{c}3.620 \\
2.841 \\
0.400 \\
15\end{array}$ & $\begin{array}{c}3.576 \\
2.946 \\
0.404 \\
15\end{array}$ \\
\hline $\begin{array}{l}O\left(N^{2.19}\right) \\
\text { heuristic of [37] }\end{array}$ & $\begin{array}{l}\max . R \\
\text { mean } R \\
\text { st. dev. } \\
\text { nb. pb. }\end{array}$ & & $\begin{array}{c}6.168 \\
3.223 \\
1.875 \\
15 \\
\end{array}$ & & $\begin{array}{c}4.758 \\
3.123 \\
0.972 \\
15 \\
\end{array}$ & $\begin{array}{c}4.838 \\
2.948 \\
0.754 \\
15 \\
\end{array}$ & $\begin{array}{c}4.127 \\
2.972 \\
0.633 \\
15 \\
\end{array}$ & $\begin{array}{c}3.703 \\
2.921 \\
0.423 \\
15 \\
\end{array}$ & $\begin{array}{c}3.666 \\
3.178 \\
0.371 \\
15 \\
\end{array}$ \\
\hline $\begin{array}{l}\text { Our relaxation scheme } \\
\text { applied to the MST: } \\
O(N \log N)\end{array}$ & $\begin{array}{l}\max . R \\
\text { mean } R \\
\text { st. dev. } \\
\text { nb. pb. }\end{array}$ & $\begin{array}{c}11.909 \\
2.727 \\
2.211 \\
4472\end{array}$ & $\begin{array}{l}9.082 \\
2.711 \\
1.515 \\
3162\end{array}$ & $\begin{array}{l}8.026 \\
2.744 \\
1.190 \\
2581\end{array}$ & $\begin{array}{l}6.088 \\
2.732 \\
1.013 \\
2236\end{array}$ & $\begin{array}{l}5.694 \\
2.715 \\
0.827 \\
1825\end{array}$ & $\begin{array}{l}5.497 \\
2.712 \\
0.738 \\
1581\end{array}$ & $\begin{array}{c}5.531 \\
2.729 \\
0.629 \\
1414\end{array}$ & $\begin{array}{c}5.207 \\
2.723 \\
0.600 \\
1290\end{array}$ \\
\hline
\end{tabular}

TABLE 4

Minimum, maximum, mean and standard deviation of the length reduction $R$ (in percents) achieved over the MST, and number of problems tested with two different approaches for the resolution of the $N$-city Steiner problem.

\begin{tabular}{|l|l|c|c|c|c|c|c|c|c|c|}
\hline & $N$ & 100 & 300 & 500 & 700 & 1000 & 3000 & 5000 & 7000 & 10000 \\
\hline & min. $R$ & 2.286 & & 2.668 & & 2.807 & & & & \\
$O\left(N^{1.317}\right)$ & max. $R$ & 3.467 & & 3.316 & & 3.283 & & & \\
heuristic & mean $R$ & 2.952 & & 3.052 & & 3.017 & & & \\
of [36] & st. dev. & 0.370 & & 0.169 & & 0.128 & & & \\
& nb. pb. & 15 & & 15 & & 15 & & & & \\
\hline & min. $R$ & 1.162 & 2.025 & 2.348 & 2.459 & 2.504 & 2.799 & 2.772 & 2.717 & 2.738 \\
Our relaxation & max. $R$ & 4.604 & 3.622 & 3.368 & 3.242 & 3.052 & 2.885 & 2.822 & 2.787 & 2.832 \\
scheme applied & mean $R$ & 2.755 & 2.757 & 2.815 & 2.803 & 2.779 & 2.842 & 2.791 & 2.762 & 2.786 \\
to the MST: & st. dev. & 0.468 & 0.266 & 0.178 & 0.180 & 0.135 & 0.036 & 0.022 & 0.024 & 0.031 \\
$O(N \log N)$ & nb. pb. & 1000 & 577 & 44 & 37 & 31 & 5 & 5 & 5 & 5 \\
\hline
\end{tabular}

largely confirmed in the following when much larger $N$ 's are considered.

When compared with the $O\left(N^{1.317}\right)$ and $O\left(N^{2.19}\right)$ heuristics of [36] and [37], it appears that our relaxation of the MST yields slightly longer suboptimal trees. Nevertheless, since our relaxation of the MST represents an $O(N \log N)$ heuristic for the Steiner problem, our approach can still trade off favorably.

The heuristic of [36] offers results that allow us to carry on the comparison above $N=50$, up to $N=10000$, as reported in Table 4. In addition, Fig. 7 represents the evolution of the maximum and mean length reduction, that we obtained with our relaxation of the MST, as a function of $N$.

The data of Table 4 and Fig. 7 show that our relaxation of the MST leads to suboptimal solution trees that keep good positive length reduction over the whole range tested, up to $N=10000$ cities. For large $N$, our results confirm over many 


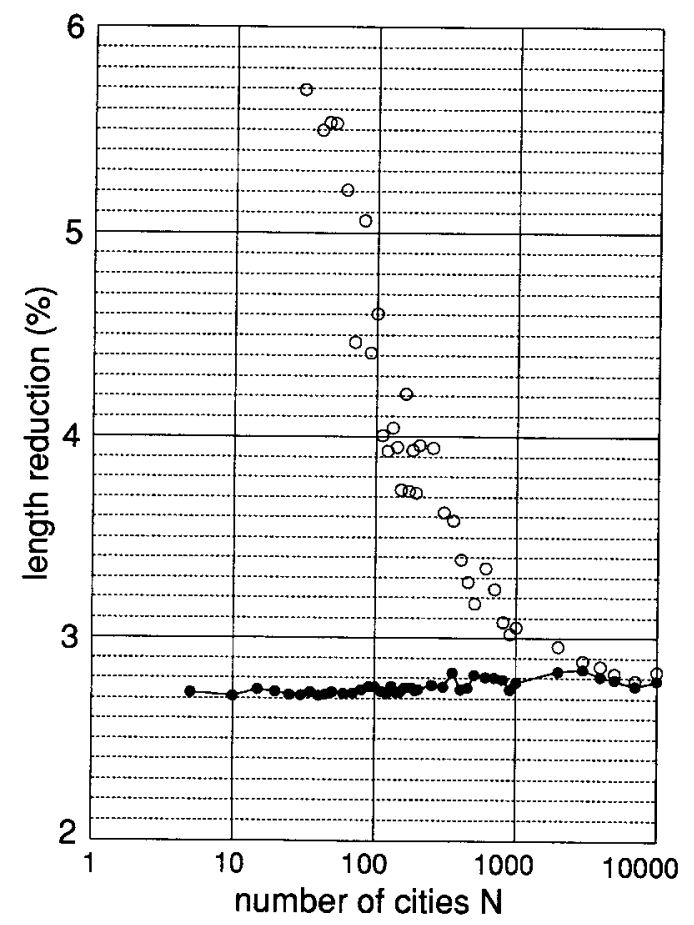

FIG. 7. Maximum (open circles) and mean (solid circles) of the length reduction $R$ (in percents) achieved by the suboptimal Steiner tree over the MST as a function of the size $N$ of the city set.

examples, after one first incursion up to $N=10000$ by [36], that trees can be found that achieve, on average, a nonvanishing length reduction over the MST. These results, as well as those of [36], further indicate that, for large $N$, the exact minimal trees of Steiner problems also achieve, on average, a nonvanishing length reduction over the MST.

With increasing $N$, our mean length reduction $R$ in Table 4 and Fig. 7 seems to stabilize to a constant value around $2.8 \%$. This is confirmed both by a maximum value of $R$ which tends to the mean of $R$ and by a standard deviation for $R$ which goes to zero with increasing $N$. In the same conditions, the heuristic of [36], although much less data are available for it, seems to display the same type of saturation for the mean $R$ but to a higher value around $3.0 \%$. This confirms the fact already observed in Table 3 that the heuristic of [36] yields shorter suboptimal trees on average but still with an algorithmic complexity higher than $O(N \log N)$.

Furthermore, the data of Table 3 reveal that the exact minimal trees (when accessible) and the suboptimal trees found by good heuristics exhibit close values for the mean length reduction. In view of this proximity of behavior, the results of Table 4 , although characterizing properties of the suboptimal trees, can serve as a basis to conjecture properties of the minimal trees of Steiner problems with large $N$. If we use our extended results of Table 4 to support the possibility of saturation of the mean length reduction $R$ for large $N$, together with the less numerous results of [36] for a better estimation of the value of this saturation, we can thus propose that the exact minimal trees for Steiner problems with large $N$ will display an average length reduction in the vicinity of $3 \%$. After the analysis of the mean length of the MST, 
as performed in Fig. 6, we can conjecture that, for large $N$, the average length of the minimal Steiner tree will be approximately $3 \%$ below $0.65 N^{1 / 2}$.

6. Discussion. The heuristic of [36] essentially considers all connected subgraphs of the MST which contain four cities, determines the minimal Steiner tree for each subgraph, which is then incorporated, through some of its Steiner points specially selected, onto the solution tree under construction. This heuristic thus performs a systematic local search for every four-city subgraph of the MST in order to discover the length reduction that is locally optimal (maximal). This heuristic is more of a classic style of combinatorics in graphs. In contrast, our relaxation algorithm closely adheres to a physical analogy that we prove fruitful. It performs length reductions in a uniform and fast way, under the sole control of surface tension forces, in a purely local manner at the level of each Steiner point and its three neighbors. This produces deformations to the tree that are fast with no systematic search of local optimality but with a global convergence to good solution trees as expected from the analogy under test. This simple and uniform procedure results in a heuristic with a low and provable complexity of $O(N \log N)$. The heuristic of [36], relatively more complicated with its systematic local search, shows a higher complexity that is only empirically estimated and, at the same time, slightly shorter solution trees.

Another interesting heuristic has been proposed for the Euclidean Steiner problem [32], [33] which is based on a simulated annealing approach [5]. Works in [35], [46], and [37] also rely, in part, on simulated annealing techniques for the resolution of various versions of the Steiner problem. The heuristic of [32] starts with a random Steiner tree. Tree transformations are implemented which consist in snipping off a randomly selected branch and, after patching the broken branch, attach this to another randomly selected branch. This results in the possibility of constructing any given tree from any other, while permanently preserving the full connectivity of the tree. These tree transformations are then accepted or rejected, depending on the change of length they entail, within the usual probabilistic scheme under the control of a temperature parameter which is gradually reduced [5]. The heuristic is tested in [32] up to $N=70$ cities. The results in [32] are presented in a way that does not allow them to fit into the comparison of Tables 3 and 4 . The quality of the solution trees in [32] is not evaluated against the MST. The scaling of the method with size $N$ of the problem is not addressed in a way that makes possible the precise determination of its algorithmic complexity. When run on an IBM 3081 computer, the best computing times reported are 17 seconds for $N=20$ and 160 seconds for $N=50$. With cities chosen uniformly at random in the square $[-10,10] \times[-10,10]$, reference [32, page 196] reports for $N=50$ a typical solution tree of length 1808.54 . When rescaled to the unit square $[0,1] \times[0,1]$ this gives a length of 90.43 , which appears well above the 4.825 mean length of the MST for $N=50$ as estimated in Table 2 and Fig. 6. Compared with our relaxation scheme that implements only local transformations to the tree at a length scale $O\left(N^{-1 / 2}\right)$, the heuristic of [32] realizes random transformations at a length scale $O(1)$ that are unable to keep the complexity below or at $O(N \log N)$ while obtaining performances comparable with ours. This is because a performant solution tree requires length adjustments at the scale $O\left(N^{-1 / 2}\right)$, and a total of at least $O\left(N^{3 / 2}\right)$ transformations involving $O(1)$-length changes are required for this goal.

Recently, another interesting approach, based on a neural network algorithm, has been proposed for the Steiner problem [38]. This neural method can be described as using a piecewise-linear curve which self-organizes to find a suboptimal tree. In the report of [38] the method, tested up to $N=100$, never performs better than Beasley's 
[36], and its solution trees have lengths that remain, on average, $1.56 \%$ above those of [36]. The complexity of the algorithm is not given explicitly in [38], and it is at least $O\left(N^{2}\right)$ since each iteration involves the calculation of a matrix of distances relative to $O(N)$ points.

Another recent heuristic for the Steiner problem is described in [39], then refined and experimentally evaluated in [40]. An interesting characteristic of this heuristic that is shared by very few of the algorithms evoked here is that it can solve Steiner problems in a space of arbitrary dimension, while our approach in its present form, as well as those, for instance, in $[22,4,31,36,37]$, is limited to the plane. The complexity of this heuristic is not explicitly established, but it is certainly above $O(N \log N)$. For Steiner problems in the plane, the evaluation in [40] is limited to $N=25$, and the best performance leads to solution trees whose average length is $2.342 \%$ below the length of the MST. With our solution trees, in the same conditions, the mean length reduction is always found above $2.710 \%$.

For the Steiner problem in the Euclidean plane, a performance guarantee is proved in [47] which states the existence of a polynomial-time heuristic that will display a performance ratio (the minimum ratio of lengths between the minimal Steiner tree and the approximation solution for the same set of cities) strictly larger than the Steiner ratio $\sqrt{3} / 2$. Reference [47], relying on the recent work of [48], also suggests a polynomial-time greedy algorithm that does not use the MST and that has the performance guarantee mentioned above. Although polynomial, the complexity of this heuristic in the plane is not given explicitly in [47], and it may be large and is certainly larger than quadratic. Also, the property that is proved in [47] does not exclude the possibility of obtaining a suboptimal tree longer than the MST for given problems. A performance guarantee with our $O(N \log N)$ heuristic relaxing the MST is the obtainment of a solution which can, at least, be made as good as this tree. Furthermore, the experimental results of Table 4 show that our algorithm was always found to converge to a solution tree strictly shorter than the initial MST.

7. Conclusion. We have presented a heuristic for the Steiner problem which is based on a physical analogy with the relaxation of a fluid film under surface tension forces. A uniform and purely local evolution scheme results for the Steiner tree, which translates into a low and provable complexity of $O(N \log N)$ for the heuristic, and allows us to tackle very large problems. The performance of this heuristic was compared with that of the best available heuristics with low complexity. Compared with [31], which represents the heuristic with the smallest complexity, our method generally leads, with the same low complexity of $O(N \log N)$, to shorter solution trees. In turn, the heuristics of [36] and [37] lead in general to solution trees slightly shorter than ours but with complexities higher than our $O(N \log N)$.

Beyond these quantitative performances we want to emphasize a specific character of our method, which is to put the Steiner problem in the more novel framework of analog relaxation of a physical type, establishing a connection with energy minimization in physical systems that revealed a fruitful analogy in other areas of optimization. In contrast, the other known algorithms with comparable performance are more of a classic style of combinatorics in graphs.

In particular, our relaxation scheme can be applied to any initial Steiner tree instead of that derived from the MST. With an initial Steiner tree randomly constructed in an $O(N)$ step, we were able to obtain solution trees achieving a positive length reduction $R$ for problem sizes up to $N \approx 100$. This type of approach can lead, for the Steiner problem, to low-complexity heuristics that do not use the MST. More 
elaborate schedules for the evolution of $T$, inspired by thermodynamic analogies and incorporating slow cooling and possibly heating phases, may also bring improvement to the performance of the fluid-film relaxation heuristic as introduced here.

\section{REFERENCES}

[1] E. N. Gilbert and H. O. Pollak, Steiner minimal trees, SIAM J. Appl. Math., 16 (1968), pp. 1-29.

[2] M. Berne and R. Graham, The shortest-network problem, Sci. Am., 260 (1989), pp. 84-89.

[3] M. R. Garey, R. L. Graham, and D. S. Johnson, The complexity of computing Steiner minimal trees, SIAM J. Appl. Math., 32 (1977), pp. 835-859.

[4] E. J. Cockayne And D. E. Hewgill, Improved computation of plane Steiner minimal trees, Algorithmica, 7 (1992), pp. 219-229.

[5] S. Kirkpatrick, C. D. Gelatt, and M. P. Vecchi, Optimization by simulated annealing, Science, 220 (1983), pp. 671-680.

[6] B. GIDAS, The Langevin equation as a global minimization algorithm, in Disordered Systems and Biological Organization, NATO ASI Series F, Vol. 20, E. Bienenstock, F. FogelmanSoulié, and G. Weisbuch, eds., Springer-Verlag, Berlin, 1986, pp. 321-326.

[7] J. Hopfield And D. W. TANK, Neural computation of decisions in optimization problems, Biological Cybernetics, 52 (1985), pp. 141-152.

[8] F. Favata And R. Walker, A study of the application of Kohonen-type neural networks to the travelling salesman problem, Biological Cybernetics, 64 (1991), pp. 463-468.

[9] R. Durbin And D. Willshaw, An analogue approach to the travelling salesman problem using an elastic net method, Nature, 326 (1987), pp. 689-691.

[10] H. Muhlenbein, M. Georges-Schleuter, and O. Kramer, Evolution algorithms in combinatorial optimization, Parallel Comput., 7 (1988), pp. 65-75.

[11] C. Peterson, Parallel distributed approaches to combinatorial optimization: Benchmark studies on the traveling salesman problem, Neural Computation, 2 (1990), pp. 261-269.

[12] C. Dussert, G. RAsigni, AND A. Llebaria, Quantization of directional properties in biological structures using the minimal spanning tree, J. Theoretical Biology, 135 (1988), pp. 295-302.

[13] A. Dress and A. Von Haeseler, eds., Trees and Hierarchical Structures, Springer-Verlag, Berlin, 1990.

[14] C. CherniaK, Local optimization of neuron arbors, Biological Cybernetics, 66 (1992), pp. 503510.

[15] R. Van De Weygaert, B. J. T. Jones, and V. J. Martinez, The minimal spanning tree as an estimator for generalized dimensions, Phys. Lett. A, 169 (1992), pp. 145-150.

[16] E. Bienenstock And R. Doursat, Elastic matching and pattern recognition in neural networks, in Neural Networks from Models to Applications, L. Personnaz and G. Dreyfus, eds., Institut pour le Développement de la Science, L'Education et la Technologie, Paris, 1989, pp. 472-482.

[17] A. L. Yuille, Generalized deformable models, statistical physics, and matching problems, Neural Computation, 2 (1990), pp. 1-24.

[18] Z. A. Melzak, On the problem of Steiner, Canad. Math. Bull., 4 (1961), pp. 143-148.

[19] E. J. Cockayne, On the Steiner problem, Canad. J. Math., 10 (1967), pp. 431-450.

[20] E. J. Cockayne, On the efficiency of the algorithm for Steiner minimal trees, SIAM J. Appl. Math., 18 (1970), pp. 150-159.

[21] W. M. Boyce, An improved program for the full Steiner tree problem, ACM Trans. Math. Software, 3 (1977), pp. 359-385.

[22] P. Winter, An algorithm for the Steiner problem in the Euclidean plane, Networks, 15 (1985), pp. 323-345.

[23] E. J. Cockayne And D. E. Hewgill, Exact computation of Steiner minimal trees in the plane, Inform. Process. Lett., 22 (1986), pp. 151-156.

[24] D. Trietsch and F. K. Hwang, An improved algorithm for Steiner trees, SIAM J. Appl. Math., 50 (1990), pp. 244-263.

[25] W. D. Sмiтh, How to find Steiner minimal trees in Euclidean d-Space, Algorithmica, 7 (1992), pp. $137-177$.

[26] F. K. Hwang and D. S. Richards, Steiner tree problems, Networks, 22 (1992), pp. 55-89.

[27] F. K. Hwang, D. S. Richards, and P. Winter, The Steiner Tree Problem, Annals of Discrete Mathematics 53, North-Holland, Amsterdam, 1992.

[28] S. K. Chang, The generation of minimal trees with a Steiner topology, J. ACM, 19 (1972), pp. 699-711. 
[29] J. Soukup, Minimum Steiner trees, roots of a polynomial and other magic, ACM/SIGMAP Newsletter, 22 (1977), pp. 37-51.

[30] P. Korhonen, An algorithm for transforming a spanning tree into a Steiner tree, in Proc. 9th Int. Math. Prog. Symp., Vol. 2, North-Holland, Amsterdam, 1979, pp. 349-357.

[31] J. M. Smith, D. T. Lee, and J. S. Liebman, An $O(n \log n)$ heuristic for Steiner minimal tree problems on the Euclidean metric, Networks, 11 (1981), pp. 23-39.

[32] M. Lundy, Application of the annealing algorithm to combinatorial problems in statistics, Biometrika, 72 (1985), pp. 191-198.

[33] M. Lundy And A. Mees, Convergence of an annealing algorithm, Math. Programming, 34 (1986), pp. 111-124.

[34] W. D. Smith, Studies in Computational Geometry Motivated by Mesh Generation, Ph.D. dissertation, Princeton University, Princeton, NJ, 1988.

[35] J. Hesser, R. Manner, And O. Stucky, Optimization of Steiner tree using genetic algorithms, in Proc. 3rd Int. Conf. Genetic Algorithms, 1989, pp. 231-236.

[36] J. E. Beasley, A heuristic for Euclidean and rectilinear Steiner problems, European J. Operational Research, 58 (1992), pp. 284-292.

[37] J. E. Beasley and F. Goffinet, A Delaunay triangulation based heuristic for the Euclidean Steiner problem, Networks, 24 (1994), pp. 215-224.

[38] Jayadeva And B. Bhaumik, A neural network for the Steiner minimal tree problem, Biological Cybernetics, 70 (1994), pp. 485-494.

[39] K. Kalpakis And A. T. Sherman, Probabilistic analysis of an enhanced partitioning algorithm for the Steiner tree problem in $R^{d}$, Networks, 24 (1994), pp. 147-159.

[40] S. Ravada AND A. T. Sherman, Experimental evaluation of a partitioning algorithm for the Steiner tree problem in $R^{2}$ and $R^{3}$, Networks, 24 (1994), pp. 409-415.

[41] M. I. Shamos and D. Hoey, Closest-point problems, in Proc. 16th Annual Symp. on Foundations of Computer Science, 1975, pp. 151-162.

[42] F. R. K. Chung and F. K. Hwang, A lower bound for the Steiner tree problem, SIAM J. Appl. Math., 34 (1978), pp. 27-36.

[43] D. Z. Du And F. K. Hwang, The Steiner Ratio Conjecture of Gilbert and Pollak Is True, Proc. Nat. Acad. Sci. U.S.A., 87 (1990), pp. 9464-9466.

[44] R. Courant and H. Robbins, What Is Mathematics?, Oxford University Press, New York, 1941.

[45] S. Wolfram, Theory and Applications of Cellular Automata, World Scientific, Singapore, 1986.

[46] K. A. Dowsland, Hill-climbing, simulated annealing and the Steiner problem in graphs, Engineering Optimization, 17 (1991), pp. 91-107.

[47] D. Z. Du, On better heuristics for Steiner minimum trees, Math. Programming, 57 (1992), pp. 193-202.

[48] A. Z. Zelikovsky, The 11/6-Approximation Algorithm for the Steiner Problem on Networks, 1992, manuscript. 